\title{
Recognition of homogeneous and heterogeneous pictures as a function of viewing context
}

\author{
MICHAEL J. KIPHART, DOUGLAS D. SJOGREN, ROSS J. LOOMIS, and HENRY A. CROSS \\ Colorado State University, Fort Collins, Colorado
}

\begin{abstract}
Although recognition memory for pictorial stimuli has been explored using either homogeneous or heterogeneous picture sets, apparently no single study has combined these stimuli and subsequently tested recognition for both types of stimuli. Subjects in two experiments were administered one of three picture sets of 100 slides. Each set consisted of homogeneous and heterogeneous slides that differed only in the ratio of homogeneous to heterogeneous stimuli: 80:20, 50:50, or 20:80. A subject's recognition memory was then examined for both types of slides. Overall recognition was high for the heterogeneous stimuli but low for the homogeneous test slides. In a signal detection analysis, the picture set involving the largest proportion of heterogeneous stimuli, 20:80, yielded the lowest recognition sensitivity to the heterogeneous stimuli. This interaction of viewing-set composition and type of test stimuli was discussed in relation to picture recognition memory research employing either homogeneous or heterogeneous stimuli.
\end{abstract}

Since the study by Shepard (1967), it has been fashionable to think of picture memory as a superior memory, and a number of investigators (Shepard, 1967; Standing, Conezio, \& Haber, 1970; Standing, 1973) have, indeed, reported recognition rates that stand in contrast to those reported for nonmeaningful materials, such as digits and the traditional nonsense syllables. However, the pictorial stimuli employed in these studies have almost always been exceedingly diverse (heterogeneous) and drawn from collections of magazine photographs that varied greatly in context and subject matter.

Goldstein and Chance (1974) argued that it is important that the nature of the pictorial stimuli be carefully described, and some investigators have utilized materials more homogeneous in nature. Howe (1967) examined recognition memory for homogeneous sets of pictures of, for example, dogs, ships, reptiles, birds, and trains and found an overall recognition rate of approximately $72 \%$, which is considerably below that reported for heterogeneous pictures. Goldstein and Chance (1970) also grouped stimuli into homogeneous sets of pictures of, for example, human faces, ink blots, and snow crystals. Faces exhibited the highest recognition (78\%); ink blots and snow crystals, however, showed only $54 \%$ and $39 \%$ recognition rates, respectively.

A recent study by Barnard, Breeding, and Cross (1984) supported the Goldstein and Chance (1974) admonition that the nature of the stimuli be considered. Barnard et al. found that the informational characteristics of exhibited objects, as revealed by cluster analysis, were important determiners of how such objects were later recognized. Overall object recognition was relatively high, but it was shown that objects low in "familiarity" did not receive

Requests for reprints should be addressed to Henry A. Cross, Department of Psychology, Colorado State University, Fort Collins, CO 80523. the disproportionate boost in recognition after a single exposure that is characteristic of unfamiliar words.

The schema that governs the organization and encoding of pictorial information is no doubt multifarious, and more research is necessary before its multidimensional nature can be appreciated. Kiphart, Sjogren, and Cross (1984) gave subjects test pictures of unusual similarity (homogeneity) and found that subsequent recognition within a signal detection framework was essentially at chance level. In the study, all of the test stimuli were homogeneous, and it was shown that the nature of the viewing context in which test slides were originally seen was relatively unimportant. In short, it made no difference whether the homogeneous test slides consisting of complex football action shots were originally seen in a homogeneous viewing set consisting of other football slides or in a heterogeneous viewing set consisting of the usual diverse assortment.

No single study has combined homogeneous and heterogeneous stimuli within the same viewing set and subsequently tested recognition for both types of test stimuli. Experiment 1 employed both types of stimuli to form three separate viewing conditions varying in the relative proportion of each stimulus type. One viewing condition consisted of 80 homogeneous and 20 heterogeneous stimuli, the second set consisted of an equal number ( 50 and 50 ) of each, and the third set was composed of 20 homogeneous stimuli and 80 heterogeneous ones. Equal numbers of homogeneous and heterogeneous slides common to the three sets were used in the test situation.

It was hypothesized that the recognition sensitivities would be greater for the heterogeneous test items than for the homogeneous ones. This particular hypothesis follows directly from the studies previously discussed. In view of the consistent finding that heterogeneous recognition rates are extremely high for 600 to 10,000 stimuli, 
it was also predicted that the heterogeneous recognition sensitivities would not differ among the three viewing conditions. With only 20 to 80 heterogeneous or homogeneous stimuli utilized in each set, the heterogeneous recognition sensitivities should be uniformly higher than the sensitivities for homogeneous stimuli across the three viewing conditions.

\section{EXPERIMENT 1}

\section{Method}

\section{Subjects and Design}

The subjects were 10 male and 41 female students from the introductory psychology course at Colorado State University. The subjects were tested individually with 17 subjects randomly assigned to each viewing condition and tested for recognition of both homogeneous and heterogeneous stimuli.

\section{Stimulus Material}

80:20 viewing condition. This condition consisted of 100 pictures: 80 homogeneous and 20 heterogeneous. Ten homogeneous and 10 heterogeneous slides were selected randomly from the 50:50 viewing condition and placed in the 80:20 viewing condition in the exact serial positions occupied in the 50:50 condition. These 20 items were later used as the signal or nondistractor items in the test set. All slides were video recorded with a 3-sec presentation and a 1-sec dark intertrial interval. The homogeneous stimuli were football action photographs selected from University files. Photographs were chosen to maximize the number of players and complexity of action. The heterogeneous stimuli were a varied assortment of buildings, mountain scenes, animals, and people.

50:50 viewing condition. The 50:50 viewing condition consisted of a total of 100 slides: 50 homogeneous and 50 heterogeneous stimuli. The slides were assigned randomly to serial positions and video recorded as in the 80:20 condition.

20:80 viewing condition. The 20:80 condition consisted of 100 stimuli: 20 homogeneous and 80 heterogeneous stimuli. The same 20 signal or nondistractor items were inserted into the same positions occupied in the 50:50 and 80:20 viewing conditions. All stimuli were video recorded as before.

Test condition. The 20 pictures originally selected from the 50:50 viewing condition were used for the test set. These 20 items were combined with 20 distractor items that included 10 new homogeneous and 10 new heterogeneous slides. The $\mathbf{4 0}$ items that constituted the test set were then ordered randomly and video recorded with a 4-sec stimulus presentation and a 2 -sec dark period between stimuli. The identical test tape was used for all three viewing conditions and for each subject. The video recordings were presented to each subject with a Sony $48-\mathrm{cm}$ (diagonal) television monitor that was mounted on the wall of a small room approximately $2 \times 3 \mathrm{~m}$.

\section{Procedure}

The subjects were seated facing the monitor and were instructed to attend carefully. The subjects were also told that the pictures would be shown quickly and consecutively and that a test would follow. After presentation of a given viewing condition, the subjects were given the test tape consisting of 20 "old" slides chosen from the original viewing set and 20 "new" slides that were not a part of the original set and were instructed to label each test slide as either "old" or "new." The subjects' responses were recorded. A debriefing statement followed. A $\mathrm{d}^{\prime}$, or sensitivity score, was calculated for both heterogeneous and homogeneous stimuli (Green \& Swets, 1966).

\section{Results and Discussion}

An analysis of variance was performed on the calculated $d^{\prime}$ scores. A significant main effect was found for test stimuli $[F(1,48)=200.65, p<.001]$, with the subjects' overall mean recognition sensitivity to the heterogeneous stimuli (3.007) being higher than that to the homogeneous stimuli (.805). A significant main effect was also observed among the three viewing conditions $(80: 20,50: 50,20: 80)$ $[\mathrm{F}(2,48)=3.76, \mathrm{p}<.05]$. The subjects in the $80: 20$ and 50:50 viewing conditions showed overall greater sensitivities than did the subjects of the $20: 80$ viewing condition. The mean sensitivities for the groups were 2.084, 2.085 , and 1.549, respectively. The $80: 20$ and 50:50 viewing conditions did not differ.

The interaction of the type of test stimulus (homogeneous vs. heterogeneous) and viewing condition (80:20, $50: 50,20: 80)$ was significant $[F(2,48)=3.85, p<.05]$ and is illustrated graphically in Figure 1 (left panel). A Newman-Keuls post hoc test revealed that the heterogeneous test slides for all three viewing conditions resulted in significantly greater sensitivities than the homogeneous stimuli. In addition, recognition sensitivities to the heterogeneous stimuli for the 80:20 and 50:50 viewing conditions were significantly greater than the sensitivities to the heterogeneous test stimuli for the 20:80 viewing condition. No other comparisons were significant.

An analysis of variance performed on the number of hits yielded two significant results. First, there was significance due to viewing conditions $[\mathrm{F}(2,48)=4.95$, $\mathrm{p}<.05]$. The means for the viewing conditions $(80: 20$, $50: 50,20: 80$ ) were $7.941,8.118$, and 6.971, respectively. A Newman-Keuls post hoc test revealed that the 20:80 group had a significantly lower number of hits than did the other two groups, which did not differ significantly. A significant effect was also noted for test stimuli $[F(1,48)$ $=15.39, \mathrm{p}<.01]$. The subjects attained fewer mean hits

EXPERIMENT 1

EXPERIMENT 2

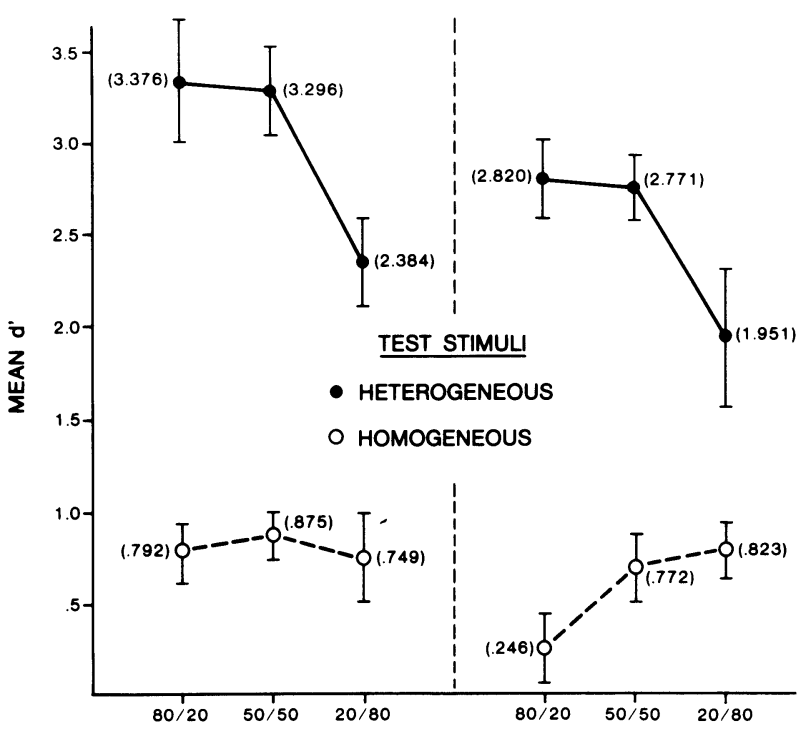

VIEWING CONDITION (HOMOGENEOUS/HETEROGENEOUS)

Figure 1. Picture recognition of homogeneous and heterogeneous stimuli as a function of the relative number of homogeneous and heterogeneous stimuli in the viewing set. Means are bounded by \pm one standard error. 
with the homogeneous (7.157) than with the heterogeneous (8.196) stimuli.

An analysis of variance was also performed on the number of false alarms, that is, the number of times individuals said "old" to a "new" stimulus. A significant main effect was noted for test stimuli $[F(1,48)=256.30$, $\mathrm{p}<.001$ ], with the subjects showing a greater mean number of false alarms to the homogeneous stimuli (4.529) than to the heterogeneous stimuli (.529).

As predicted, there was a large difference in the ability of subjects to recognize homogeneous and heterogeneous stimuli. In all three viewing conditions, the heterogeneous slides yielded high recognition rates that sharply contrasted with the near-chance performance of the subjects to homogeneous stimuli. Performance in the homogeneous test was similar to that previously reported (Kiphart et al., 1984) and demonstrated, again, that under some conditions picture memory could be poor.

The depression of the 20:80 group in the heterogeneous test was counter to the original prediction; for this reason, it was decided that Experiment 1 should be replicated.

\section{EXPERIMENT 2}

\section{Method}

\begin{abstract}
Subjects and Design
The subjects were 10 male and 21 female students from the introductory psychology course at Colorado State University. The subjects were tested individually and were assigned to one of the three viewing contexts: 80:20, 50:50, and 20:80. All subjects were tested for recognition of both homogeneous and heterogeneous stimuli. The stimuli used and the procedure followed were the same as those of Experiment 1.
\end{abstract}

\section{Results}

An analysis of variance was performed on the calculated $d^{\prime}$ scores. No significant effect was found for viewing condition $[F(2,28)=1.21, p<.05]$. However, the means did follow a pattern similar to that exhibited in Experiment 1 , with the 80:20, 50:50, and 20:80 viewingcondition means equal to $1.533,1.771$, and 1.387 , respectively. A significant main effect was observed for test stimuli $[F(1,28)=15.48, p<.001]$, with the subjects showing an overall higher mean sensitivity to the heterogeneous stimuli $(2.575)$ than to the homogeneous stimuli (.615).

The interaction of viewing condition $(80: 20,50: 50$, $20: 80$ ) with the type of test stimuli (homogeneous, heterogeneous) was significant $[F(2,28)=5.64, p<.05]$ and is illustrated in Figure 1 (right panel). A Newman-Keuls post hoc test revealed that the heterogeneous test stimuli for all three viewing conditions resulted in significantly greater mean sensitivities than the homogeneous stimuli. In addition, the recognition sensitivities to the heterogeneous stimuli for 80:20 and 50:50 viewing conditions were significantly greater than the mean sensitivity for the $20: 80$ viewing condition.
An analysis of variance performed on the number of hits failed to reveal significance. However, an analysis of variance conducted on the number of false alarms was significant. A main effect was noted for test stimuli $[\mathrm{F}(2,28)=145.59, \mathrm{p}<.001]$, with the subjects showing a greater overall mean number of false alarms to the homogeneous test stimuli (4.985) than to the heterogeneous test stimuli $(0.710)$. The interaction of test stimuli and viewing condition was also significant for false alarms $[F(2,28)=4.05, p<.05]$. This interaction was the result of a decrease in mean false alarms $(5.7,4.6$, and 4.5 for homogeneous test slides across viewing conditions), which was accompanied by a mean increase of false alarms $(0.20,0.69$, and 1.37 for the heterogeneous test stimuli across the same viewing condition, 80:20, 50:50, and 20:80, respectively).

\section{GENERAL DISCUSSION}

In two identical studies, it was shown that picture recognition is not a superior memory when the viewing and test stimuli are exceedingly homogeneous. In fact, under the present conditions, the performance with homogeneous test stimuli was near chance, a finding that parallels that reported by Kiphart et al. (1984) for the same materials. The fact that in both instances the pictures were real scenes indicates that one does not have to resort to random shapes or other artificial stimuli to reduce recognition to a low level. By contrast, recognition performance with the more typical heterogeneous stimuli employed in this domain was high. One must agree with Loftus (1982) that it is inappropriate to conclude that picture memory is an exceptional memory with rules that bear little or no similarity to those that apply in verbal recognition. The sensitivities for homogeneous stimuli suggest that not all picture memory tasks are superior and that they can sometimes be close to chance level. On the other hand, the finding (Barnard et al., 1984) that the judged characteristics of objects may influence how they are subsequently recognized, in a way different from words, should provide a caution against the acceptance of picture recognition as simply as extension of the principles of verbal recognition memory. Horton and Mills (1984), in their review, maintained that the current evidence is that pictures and words are dealt with differently in memory even though this fact may not preclude a single memory system.

A finding in both experiments that is not easily explained is that recognition memory for a relatively small set of heterogeneous slides can be significantly reduced with the introduction of a few (20\%) homogeneous slides into the original viewing set. One could speculate that the subject, knowing only that a test is to follow, is alerted to give special attention to those homogeneous stimuli that are demarcated by their common class and infrequent occurrence and that this results in an insufficient allocation of attentional resources to the heterogeneous stimuli. This speculation is supported by the interaction between test stimuli and viewing conditions for false alarms. False alarms increased across viewing conditions $(80: 20,50: 50,20: 80)$ for heterogeneous stimuli and decreased across viewing conditions for homogeneous stimuli. In summary, the number and direction of false alarms support the proposition that an insufficient allocation of attentional resources was applied to the heterogeneous stimuli. The difficulty with this conjecture is that it suggests improved recognition for the homogeneous stimuli, which presumably receive the increased attention. Clearly, this was not the case. Unless one argues further that the homogeneous recognition task in this particular study was simply too difficult to benefit by an increased allocation of attentional resources, the explanation given here seems inadequate. Nevertheless, the empirical finding observed in both studies should be explored. Any such minor manipulation of the viewing set that significantly reduces recognition memory for heterogeneous pictures deserves systematic examination. 


\section{REFERENCES}

Barnard, W. A., Breeding, M., \& Cross, H. A. (1984). Object recognition as a function of stimulus characteristics. Bulletin of the Psychomonic Society, 22, 14-18.

Goldstein, A. G., \& CHANCE, J. E. (1970). Visual recognition memory for complex configurations. Perception \& Psychophysics, 9, 237-241.

Goldstein, A. G., \& Chance, J. E. (1974). Some factors in picture recognition memory. Journal of General Psychology, 90, 69-85.

Green, D. M., \& SwETs, J. A. (1966). Signal detection theory and psychophysics. New York: Wiley.

HoRToN, D. L., \& Milss, C. B. (1984). Human learning and memory. Annual Review of Psychology, 35, 361-394.

HowE, M. J. A. (1967). Recognition for photographs in homogeneous sequences. Perceptual and Motor Skills, 24, 1181-1182.

KiphaRT, M. J., SJogren, D. D., \& Cross, H. A. (1984). Some fac- tors involved in complex picture recognition. Bulletin of the Psychonomic Society, 22, 197-199.

LofTus, G. R. (1982). Picture memory methodology. In C. R. Puff (Ed.), Research methods in human memory and cognition. New York: Academic Press.

SHEPARD, R. (1967). Recognition memory for words, sentences, and pictures. Journal of Verbal Learning and Verbal Behavior, 6, 156-163.

STANDING, L. (1973). Learning 10,000 pictures. Quarterly Journal of Experiment Psychology, 25, 207-222.

Standing, L., Conezio, H., \& Haber, R. N. (1970). Perception and memory for pictures: Single-trial learning of 2,500 visual stimuli. Psychonomic Science, 19, 73-74.

(Manuscript received for publication September 24, 1984.) 